\title{
Robust flatness-based switching reconfiguration control using state flow machines of electronic throttle valve
}

\author{
Hajer Gharsallaoui ${ }^{1, a}$, Wafa Gritli ${ }^{1}$, and Mohamed Benrejeb ${ }^{1}$ \\ LA.R.A Automatique, Ecole Nationale d'Ingénieurs de Tunis, Université de Tunis El Manar, BP 37, Le Belvédère, 1002 \\ Tunis, Tunisie.
}

\begin{abstract}
In this paper, a robust Fault Tolerant Control reconfiguration approach using State Flow Machines is proposed. Indeed, this reconfiguration strategy is based on robust flatness-based switching control using state machines and flow charts. This approach is developed in discrete time framework in order to track a reference trajectory starting from a flat output variable. For each model, a corresponding flatness-based controller is designed and consequently, a multi controller structure is obtained. The switching flatness- based control is based on switching between identified Operating Modes $(\mathrm{OM})$ using state flow machines. The Luenberger observer's gains are determined using LMIs tools in order to identify the corresponding OM. The localization of the current $\mathrm{OM}$ is carried out by minimization of a performance test characterizing the distance between the system and the given OM. Study of the stability as well as the use of anti-windup devices related to switching between controllers have been considered in the proposed approach. The proposed approach is applied to the nonlinear system which is in our case of study an Electronic Throttle Valve (ETV) using state flow machines modeling.
\end{abstract}

\section{Introduction}

General multiple model-based control approaches have been developed in last decade. Thus, in the literature, there are many strategies that take in consideration the multi-models methods used for reconfiguration of control law, [1], [2] and [3]. Furthermore, Multiple Models (MM) method is proposed in [4]. In addition, controller switching approach represents a class of active fault tolerant control detailed in [5]. Moreover, Multiple Models Switching and Tuning (MMST) approach that concerns more particularly the reconfigurable control method is proposed in [3], [6] and [7]. In this paper, for a nonlinear process with multiple OMs, a robust control reconfiguration strategy, based on switching control, is used to ensure stability and desired performances. The LMI tools which are based on quadratic Lyapunov criteria are used in order to guaranty the stability of the global system. The performances obtained by switching in terms of tracking trajectory and fault rejection are discussed in this paper and the stability of the corresponding switched discrete-time linear systems is given. The case of ETV is studied.

\section{Reconfiguration fault tolerant control approach}

\subsection{Description}

A robust RST multicontroller based on flatness is proposed. The controller selection strategy is based on switching between identified Operating Modes (OMs). A stateflow is proposed in order to select the identified controller. The localization of the current $\mathrm{OM}$ is carried out by minimization of a performance test characterizing the distance between the system and the given OM.

\subsection{Stability analysis}

The stability analysis of a switching control strategy consists on two steps, [1]

- stability analysis of each subsystem, i.e., each controller must asymptotically stabilize each process operating mode,

- stability analysis of the overall system for arbitrary switching signals.

To guarantee the stability of the systems, Linear Matrix Inequalities, represented by 1 must be solved [8].

$$
\left(\begin{array}{cc}
P & P A_{j}-Y_{j} C_{j} \\
\left(P A_{j}-Y_{j} C_{j}\right)^{T} & P
\end{array}\right)>0
$$

with $P$ a symmetric positive definite matrix given by $L_{j}=$ $P^{-1} Y_{j}$ where $L_{j} \in \mathbb{R}^{n \times m}$ is the Lunberger observer gain for the $j^{\text {th }}$ model.

\section{RST multicontroller design based on flatness}

\subsection{RST multicontroller structure}

Let us denote the open-loop discrete-time process transfer function by

$$
H_{j}\left(q^{-1}\right)=\frac{B_{j}\left(q^{-1}\right)}{A_{j}\left(q^{-1}\right)}
$$


$A_{j}(q)$ and $B_{j}(q)$ are polynomials defined by (3) and (4) where the parameters $a_{j, i}$ and $b_{j, i}$ are constants, $j=1, \ldots, m$, $i=0,1, \ldots, n-1$ and $q^{-1}$ is the causal operator.

$$
\begin{gathered}
A_{j}\left(q^{-1}\right)=1+a_{j, n-1} q^{-1}+\ldots+a_{j, 1} q^{-n+1}+a_{j, 0} q^{-n} \\
B_{j}\left(q^{-1}\right)=b_{j, n-1} q^{-1}+\ldots+b_{j, 1} q^{-n+1}+b_{j, 0} q^{-n}
\end{gathered}
$$

The realization of an RST controller based on flatness is carried out by considering the method of direct calculation of the state vector $Z_{j, k}=\left(\begin{array}{llll}z_{j, k} & z_{j, k+1} & \ldots & z_{j, k+n-1}\end{array}\right)^{T}$, [9].

The state space representation of the system in its controllable form is such that

$$
\left\{\begin{array}{l}
Z_{j, k+1}=\mathbf{A}_{j} Z_{j, k}+\mathbf{B}_{j} u_{j, k} \\
y_{j, k}=\mathbf{C}_{j} Z_{k}
\end{array}\right.
$$

where the matrix $\mathbf{A}_{j}, \mathbf{B}_{j}$ and $\mathbf{C}_{j}$ are given by

$$
\begin{gathered}
\mathbf{A}_{j}=\left(\begin{array}{ccccc}
0 & 1 & 0 & \cdots & 0 \\
0 & 0 & 1 & \ddots & \vdots \\
\vdots & \ddots & \ddots & \ddots & 0 \\
0 & \cdots & 0 & 0 & 1 \\
-a_{j, 0}-a_{j, 1} & \cdots & -a_{j, n-2} & -a_{j, n-1}
\end{array}\right) \\
\mathbf{B}_{j}=\left(\begin{array}{llll}
0 & \cdots & 0 & 1
\end{array}\right)^{T} \\
\mathbf{C}_{j}=\left(b_{j, 0} b_{j, 1} \cdots b_{j, n-1}\right)
\end{gathered}
$$

The flatness control law is given by the following relation

$$
u_{k, j}=K(q) z_{k, j}^{d}+(a-k) Z_{k, j}
$$

where $a$ and $k$ are two constant vectors constituted by the $a_{j, i}$ and $k_{i}$ coefficients of the $A_{j}(q)$ and $K(q)$ polynomials given by

$$
a=\left(\begin{array}{llll}
a_{j, 0} & a_{j, 1} & \cdots & a_{j, n-1}
\end{array}\right), k=\left(\begin{array}{llll}
k_{0} & k_{1} & \cdots & k_{n-1}
\end{array}\right)
$$

The polynomial $K(q)$ is the denominator of the tracking dynamics which is a discrete-time $n^{\text {th }}$ order model equivalent of a continuous-time one composed of second order with fixed damping factor $\xi$, fixed natural frequency $\omega_{0}$, given by (11), and a first order model with a fixed time constant $\tau$, given by (12), where $s$ is the Laplace operator.

$$
\begin{gathered}
\frac{\omega_{0}^{2}}{s^{2}+2 \omega_{0} \xi s+\omega_{0}^{2}} \\
\frac{1}{1+\tau s}
\end{gathered}
$$

The structure of RST controller can be then obtained by

$$
S_{j}\left(q^{-1}\right) u_{k}=K(q) z_{j, k}^{d}-R_{j}\left(q^{-1}\right) y_{j, k}
$$

with

$$
R_{j}\left(q^{-1}\right)=(k-a) \mathbf{A}_{j}^{n-1} O_{\left(\mathbf{A}_{j}, \mathbf{C}_{j}\right)}^{-1} Q
$$

$S_{j}\left(q^{-1}\right)=1+(a-k)\left(\mathbf{A}_{j}^{n-1} O_{\left(\mathbf{A}_{j}, \mathbf{C}_{j}\right)}^{-1} M_{\left(\mathbf{A}_{j}, \mathbf{B}_{j}, \mathbf{C}_{j}\right)}-\left(\mathbf{A}_{j}{ }^{n-2} \mathbf{B}_{j} \cdots \mathbf{B}_{j}\right)\right) Q^{*}$

and

$$
\begin{aligned}
& Q=\left(q^{-(n-1)} q^{-(n-2)} \cdots q^{-1} 1\right)^{T} \\
& Q^{*}=\left(q^{-(n-1)} q^{-(n-2)} \cdots q^{-1}\right)^{T}
\end{aligned}
$$

$M_{\left(\mathbf{A}_{j}, \mathbf{B}_{j}, \mathbf{C}_{j}\right)}$ and $O_{\left(\mathbf{A}_{j}, \mathbf{C}_{j}\right)}$ are the controllability and the observability matrices.

\subsection{Trajectories planning}

The open loop control law can be determined by the following relations [10].

$$
\begin{gathered}
u_{j}^{d}(t)=f\left(z_{j}^{d}(t), \ldots, z_{j}^{d(r+1)}(t)\right) \\
y_{j}^{d}(t)=g\left(z_{j}^{d}(t), \ldots, z_{j}^{d(r)}(t)\right)
\end{gathered}
$$

where $f$ and $g$ are vectorial functions. Then, it is sufficient to find a desired continuous flat trajectory $t \mapsto z_{j}^{d}(t)$ that must to be differentiable at the $(r+1)$ order.

In order to plan the desired flat trajectory $z_{j}^{d}(t)$, the polynomial interpolation technique is used. Let consider the state vector $Z_{j}^{d}(t)=\left(z_{j}^{d}(t) \dot{z}_{j}^{d}(t) \ldots z_{j}^{d(r+1)}(t)\right)^{T}$ containing the desired continuous flat output and its successive derivatives. $t_{0}$ and $t_{f}$ are the two moments known in advance. The expression of $Z_{j}^{d}(t)$ can be given as following [10]

$$
Z_{j}^{d}(t)=M_{j, 1}\left(t-t_{0}\right) c_{j, 1}\left(t_{0}\right)+M_{j, 2}\left(t-t_{0}\right) c_{j, 2}\left(t_{0}, t_{f}\right)
$$

where $M_{j, 1}$ and $M_{j, 2}$ are such as

$$
M_{j, 1}=\left(\begin{array}{cccc}
1 & t & \cdots & \frac{t^{n-1}}{(n-1) !} \\
0 & 1 & \cdots & \frac{t^{(n-2)}}{(n-2) !} \\
\vdots & \ddots & \ddots & \vdots \\
0 & \cdots & 0 & 1
\end{array}\right)
$$

$$
M_{j, 2}=\left(\begin{array}{cccc}
\frac{t^{n}}{n !} & \frac{t^{n+1}}{(n+1) !} & \cdots & \frac{t^{2 n-1}}{(2 n-1) !} \\
\frac{t^{n-1}}{(n-1) !} & \frac{t^{n}}{n !} & \cdots & \frac{t^{(n-2)}}{(n-2) !} \\
\vdots & \ddots & \ddots & \vdots \\
t & \cdots & \frac{t^{n-1}}{(n-1) !} & \frac{t^{n}}{n !}
\end{array}\right)
$$

and the vectors $c_{j, 1}$ and $c_{j, 2}$ defined by

$$
\begin{gathered}
c_{j, 1}=Z_{j}^{d}\left(t_{0}\right) \\
c_{j, 2}=M_{j, 2}^{-1}\left(t_{f}-t_{0}\right)\left(Z_{j^{d}}\left(t_{f}\right)-M_{j, 1}\left(t_{f}-t_{0}\right) Z_{j}^{d}\left(t_{0}\right)\right)
\end{gathered}
$$

After planning a desired flat trajectory, the output desired trajectory $y_{j, k}^{d}$ is defined. In the discrete-time framework, the real output $y_{j, k}$ have asymptotically to track this such as [25], [10].

$$
y_{j, k}^{d}=B_{j}(q) z_{j, k}^{d}
$$

\section{Application to the control of an electronic throttle valve}

\subsection{Electronic throttle valve modelling}

The case of the Electronic Throttle Valve (ETV), of the Fig. 1. is studied to test the proposed controller.

The electrical part is modeled by 26.

$$
u=L \frac{d i}{d t}+R i+E, E=k \frac{d \theta_{m}}{d t}=k \omega
$$




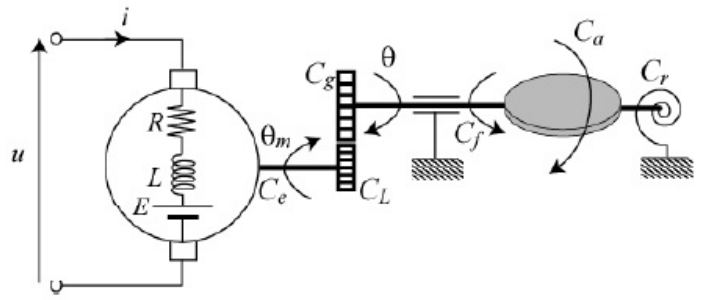

Fig. 1. Electronic throttle system [11]

where $L$ is the inductance, $R$ the resistance, $E$ the electromotive force of its armature, $u$ and $i$ the voltage and the armature current respectively, $k$ an electromotive force constant, $\theta_{m}$ the position of the motor shaft and $\omega$ the angular velocity of the motor rotor [12] and [13].

The mechanical part of the throttle is modeled by a gear reducer characterized by its reduction ratio $\eta$ such as (27)

$$
\eta=\frac{\theta_{m}}{\theta}=\frac{C_{g}}{C_{L}}
$$

where $C_{L}$ is the load torque and $C_{g}$ the gear torque.

The mechanical part is modeled according to 28 , such that, [12] and [13].

$$
J \frac{d \Omega}{d t}=C_{e}-C_{f}-C_{r}-C_{a}
$$

$J$ is the overall moment of inertia, $\Omega=\frac{d \theta}{d t}$ the angular velocity of the throttle valve, $C_{e}=k_{e} i$ the electrical torque where $k_{e}$ a constant, $C_{f}$ the torque caused by mechanical friction, $C_{r}$ the spring resistive torque and $C_{a}$ the torque generated by the airflow.

By substituting in equation (28), the expressions $C_{e}, C_{f}$ and $C_{r}$ and by neglecting the torque generated by the airflow $C_{a}$, the transfer function of the model becomes such as $(29)$

$$
H(s)=\frac{(180 / \pi) k_{e} / l}{J L s^{3}+J R s^{2}+\left(k k_{e}+k_{s} L\right) s+R k_{s}}
$$

with $k_{s}=\left(180 / \pi / l^{2}\right) k_{r}, l$ a constant and $s$ the Laplace operator.

The identified parameters of $H(s)$ are given in Table 1

Table 1. Model's parameters

\begin{tabular}{|c|c|}
\hline Parameters & Values \\
\hline$R(\Omega)$ & 2.8 \\
\hline$L(H)$ & 0.0011 \\
\hline$k_{e}(\mathrm{~N} . \mathrm{m} / \mathrm{A})$ & 0.0183 \\
\hline$k_{r}(\mathrm{v} / \mathrm{rad} / \mathrm{s})$ & 0.0183 \\
\hline$J\left(\mathrm{~kg} . \mathrm{m}^{2}\right)$ & $4 \times 10^{-6}$ \\
\hline$l$ & 16.95 \\
\hline
\end{tabular}

The default position $\theta_{0}$ of the ETV creates a discontinuity in the dynamics of the system. The operating mode depends on the throttle position. Two linear models $(j=1,2)$ have been chosen to carry out the study with the sampling period; $T_{e}=0.002 \mathrm{~s}$.
- A model representing the position of the plate above the position by default with $k_{s}=1.877 \times 10^{-4} \mathrm{~kg} \cdot \mathrm{m}^{2}$. The corresponding discrete-time transfer function $H_{1}\left(q^{-1}\right)$ is given by 30 ).

$$
H_{1}\left(q^{-1}\right)=\frac{0.007833 q^{-1}+0.01396 q^{-2}+0.0007724 q^{-3}}{1-1.948 q^{-1}+0.954 q^{-2}-0.006152 q^{-3}}
$$

- A model representing the position of the plate below the position by default with $k_{s}=1.384 \times 10^{-3} \mathrm{~kg} \cdot \mathrm{m}^{2}$. The corresponding discrete-time transfer function $\mathrm{H}_{2}\left(q^{-1}\right)$ is given by 31 .

$$
H_{2}\left(q^{-1}\right)=\frac{0.007832 q^{-1}+0.01396 q^{-2}+0.0007724 q^{-3}}{1-1.946 q^{-1}+0.954 q^{-2}-0.006152 q^{-3}}
$$

\subsection{RST multicontroller based on flatness}

The dynamics chosen are those of a fourth-order continuoustime system with a damping factor $\xi=0.8$, a natural frequency $\omega_{0}=20 \mathrm{rad} / \mathrm{s}$ and a time constant $\tau=0.1 \mathrm{~s}$. The polynomial $K(q)$ is then given by (32).

$$
K(q)=q^{4}-3.897 q^{3}+5.695 q^{2}-3.699 q+0.9012
$$

The following polynomials were obtained for the RST multicontroller

$R_{1}\left(q^{-1}\right)=-0.009138+0.06373 q^{-1}-0.09832 q^{-2}+0.04374 q^{-3}$

$$
R_{2}\left(q^{-1}\right)=-0.03525+0.1149 q^{-1}-0.1236 q^{-2}+0.0439 q^{-3}
$$

$S_{1}\left(q^{-1}\right)=1-0.9495 q^{-1}-0.005626 q^{-2}-7.058 \times 10^{-6} q^{-3}$

$S_{2}\left(q^{-1}\right)=1-0.9505 q^{-1}-0.006031 q^{-2}-2.722 \times 10^{-5} q^{-3}$

\subsection{Stability analysis}

The Luenberger observers's gains are given by (37).

$$
L_{1}=\left(\begin{array}{l}
-24.5159 \\
-59.3827 \\
-105.0460
\end{array}\right) ; \quad L_{2}=\left(\begin{array}{l}
-24.5175 \\
-59.3817 \\
-105.0352
\end{array}\right)
$$

The combination of the two controllers with the two process transfer functions, is found to be globally asymptotically stable with $P$ definite positive, is given by (38).

$$
P=\left(\begin{array}{ccc}
0.1839 & -0.1798 & 0.0587 \\
-0.1798 & 0.1759 & -0.0574 \\
0.0587 & -0.0574 & 0.0188
\end{array}\right)
$$

\subsection{Simulation results}

The desired trajectories $z_{1}^{d}(t)$ and $z_{2}{ }^{d}(t)$ are given by Fig. 2

The simulation results are given in Fig. 3, 4 and 5, This figures show the effectiveness of the RST multicontroller based on flatness in terms of tracking a desired trajectory with high performance and disturbances rejection. 


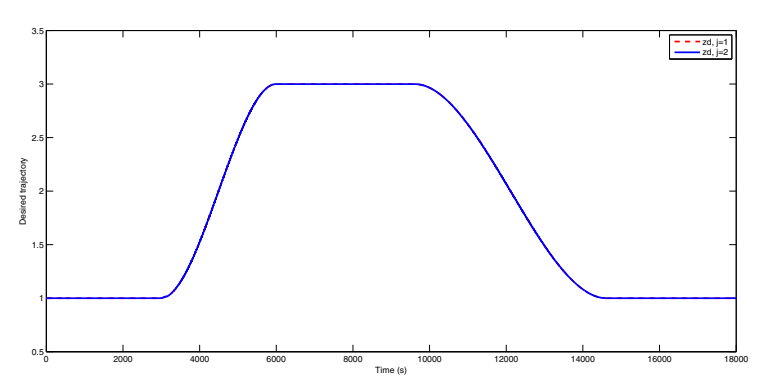

Fig. 2. Desired trajectory $z_{j}{ }^{d}$

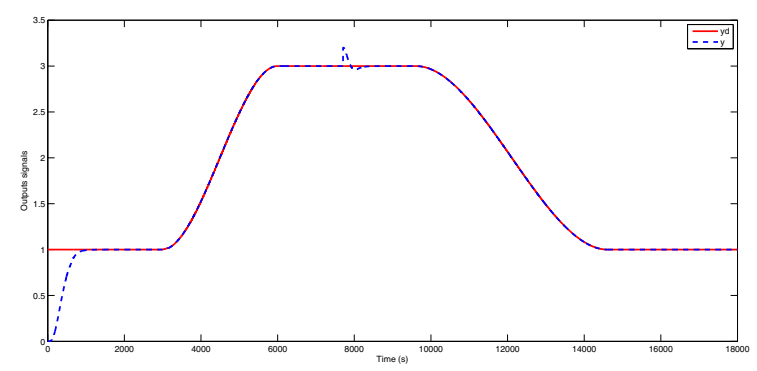

Fig. 3. System output $y$ and desired output $y^{d}$

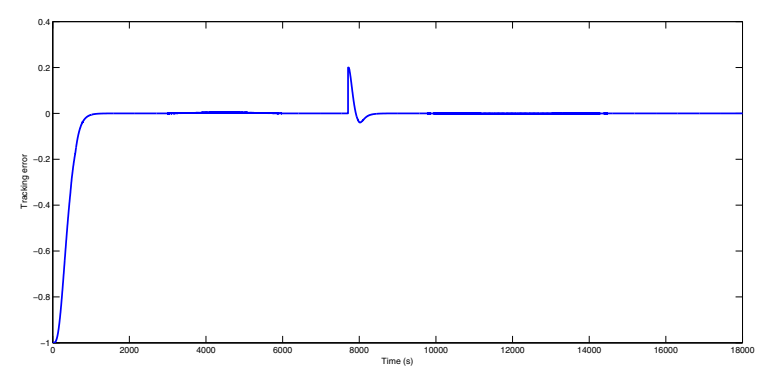

Fig. 4. Tracking error

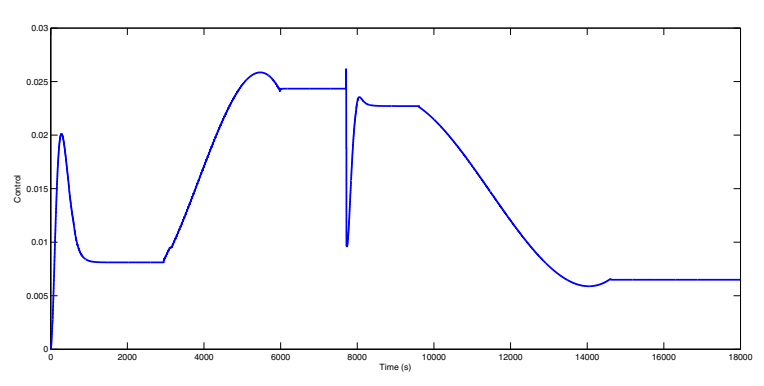

Fig. 5. Control

\section{Conclusion}

The proposed approach based on the flatness is applied to an Electronic Throttle Valve (ETV) with multiple Operating Modes (OMs). For each model, a corresponding RST controller based on flatness is designed and consequently, a reconfiguration strategy based on multi-controllers structure obtained. The switching between identified models of an ETV was based on the minimization of a performance test characterizing the distance between the system and the given OM. Simulation results proved the effectiveness of the proposed multicontroller.

\section{References}

1. P. Charbonnaud, J.F. Carrilo and S. Médar, Robust control reconfiguration of a thermal process with multiple operating modes, IEEE transactions on control systems technology, pp. 529-538, 2003.

2. S. Médar, P. Charbonnaud and F. Noureddine, Active fault accommodation of a three tank system via switching control, IFAC World Congress, Barcelona, 2002.

3. K. S. Narendra, O. A. Driollet, M. Feiler and K. George, Adaptive control using multiple models, swtiching and tuning, International Journal of Adaptative Control and Signal Processing, pp. 87-102, 2003.

4. J. Lemos, L. Ratos and J. Marques, Switching reconfigurable control based on hidden markov models, American Control Conference, San Diego, 1999.

5. M. Mahmoud, J. Jiang and Y. Zhang, Stochastic stability analysis of fault tolerant control systems in the presence of noise, IEEE Transactions on Automatic Control, pp. 1810-1815, 2001.

6. M. Blanke, M. Kinnaert,J. Lunze and M. Staroswiecki, Diagnosis and Fault-Tolerant Control, Springer, London, 2006.

7. Y. Zhang and J. Jiang, Bibliographical review on reconfigurable fault-tolerant control systems, Proceeding of the SAFEPROCESS: 5th IFAC Symposium on Detection and Safety for Technical Processes, Washington, 2003.

8. L. ElGhaoui and S. Niculescu, Advances in Linear Matrix Inequality methods in control, Philadelphia, 2000.

9. F. Rotella, F. J. Carrilo and M. Ayadi, Digital flatnessbased robust controller applied to a thermal process, in Proceedings of the 2001 IEEE International Conference on Control Applications (CCA '01), pp. 936-941, Mexico City, 2001.

10. H. Gharsallaoui, M. Ayadi, M. Benrejeb and P. Borne, Flatness-based Control and Conventional RST Polynomial Control of a Thermal Process, in International Journal of Computers, Communications and Control (IJCCC), vol. 4, pp. 41-56, 2009.

11. I. Aidi, M. Ayadi, M. Benrejeb and P. Borne, Flatnessbased Control of Throttle Valve Using Neural Observer, in International Journal of Research and Surveys, vol. 12, pp. 333-344, 2012.

12. M. Vasak, M. Baotic, I. Petrovic and N. Peric, Hybrid Theory-Based Time-Optimal Control of an Electronic Throttle, in IEEE Transactions on Industrial Electronics, vol. 54, pp. 1483-1494, 2007.

13. M. Lebbal, H. Chafouk, G. Hoblos and D. Lefebvre, Modelling and Identification of Non-Linear Systems by a Multimodel Approach: Application to a Throttle Valve, in International Journal Information and Systems Scienc, vol. 3, pp. 67-87, 2007. 